\title{
Assessment of Somatic Support Process for Pregnant Brain Death Patients Occurring in a Transition Country Between Asia and Europe from Medical, Ethical, Legal and Religious Aspects
}

\author{
Ömer Faruk Boran ${ }^{1}$ (D) Fatih Mehmet Yazar $^{2}$ (D) Murat Bakacak ${ }^{3}$ (D) \\ Dilek Soylu ${ }^{4}$ (D) Nurullah Yazar ${ }^{5}$ (D) Hafize Öksüz ${ }^{1}$ (D)
}

Published online: 27 November 2019

(c) Springer Science+Business Media, LLC, part of Springer Nature 2019

\begin{abstract}
In spite of the fact that brain death during pregnancy is not a common occurrence, it is an important ethical problem for all cultures and religions can have a significant influence on the donation decision after brain death. Therefore, this study aimed to present the case of a pregnant patient developing brain death which occurred in our intensive care unit and to compare the medical, ethical and legal problems relating to pregnant cases developing brain death with 24 cases in the literature. A 21-yearold 19-week pregnant case with gestational diabetes was monitored in the anesthesia intensive care unit and developed brain death due to intracranial mass and intraventricular hemorrhage. Though brain death is a situation well understood by organ transplant professionals, brain death developing in pregnant patients still involves many medical, ethical and legal problems.
\end{abstract}

Keywords Brain death in pregnancy · Donation decision · Muslim families

\section{Introduction}

Brain death (BD), which was first described by Mollaret and Goulon (1959), refers to irreversible loss of brain stem functions. Support of patients with BD diagnosis is unnecessary apart from some situations like the patient being an organ donor and pregnancy (Esmaeilzadeh et al. 2010). Though support of patients developing BD who are organ donors until the organ transplant procedure is a common situation encountered by organ transplant professionals, the support of pregnant patients developing BD until the birth of the baby is an extreme situation (Esmaeilzadeh et al. 2010; Wawrzyniak 2015; Gopčević et al. 2017). Since 1982, 24 patients with

Ömer Faruk Boran

ofboran@ksu.edu.tr

Extended author information available on the last page of the article 
brain death in pregnancy have been reported in the literature (Dillon et al. 1982; Heikkinen et al. 1985; Field et al. 1988; Bernstein et al. 1989; Antonini et al. 1992; Anstötz 1993; Nettina et al. 1993; Béguin 1993; Wuermeling 1994; Iriye et al. 1995; Vives et al. 1996; Catanzarite et al. 1997; Lewis and Vidovich 1997; Beca et al. 1998; Spike 1999; Lane et al. 2004; Hussein et al. 2006; Souza et al. 2006; Mejia et al. 2008; Yeung et al. 2008; Said et al. 2013; Wawrzyniak 2015; Gopčević et al. 2017; Holliday and Magnuson-Woodward 2017). However, with the increase in awareness and necessity of organ transplantation, the number of applications of BD procedures in intensive care units has increased, with a resulting increase in both 24 pregnant BD cases (Table 1). Both globally and in our country, there is no consensus about what should be done for pregnant cases developing BD, with no serious awareness and no legal regulations in many countries. Turkey is a developing country acting as a bridge between Asia and Europe. Though accepting a laic form of government for nearly a century, many people still attach importance to the opinions of religious authorities in daily life and decisions they make. In the literature, brain death cases occurring in pregnancy generally are found in socioeconomically developed Western societies, with only medical, ethical and legal aspects assessed (Bernstein et al. 1989; Vives et al. 1996; Suddaby et al. 1998; Souza et al. 2006; Yeung et al. 2008; Esmaeilzadeh et al. 2010; Wawrzyniak 2015). For the first time in the literature, our study emphasizes how to assess the event in religious terms. We think it will be beneficial to present this type of rare case in terms of increasing awareness about the necessary interventions for these patients, especially, and about the medical, ethical and legal problems.

Here we present our experience of a pregnant patient developing BD in the intensive care unit and compare with 24 cases in the literature.

\section{Methods}

In this study, we present the case of a pregnant patient developing BD in intensive care. Additionally, pregnant BD cases were screened from the English literature using PubMed and Google Scholar. During screening, the key words of pregnant, pregnancy, brain death and organ transplant were used. Screening encompassed 1982 to August 2018. The year of publication, country, maternal age, gestational age of fetus when brain death was diagnosed, duration until birth and neonatal outcomes were recorded along with demographic and technical data relating to the mother and fetus. Inclusion criteria for publications obtained from screening included patient developing BD while pregnant and administration of somatic support until fetus maturity after patients developed BD. If publications did not include somatic support after development of BD or if the fetus was mature when BD developed and Cesarean was immediately performed, publications were not included in the assessment. 


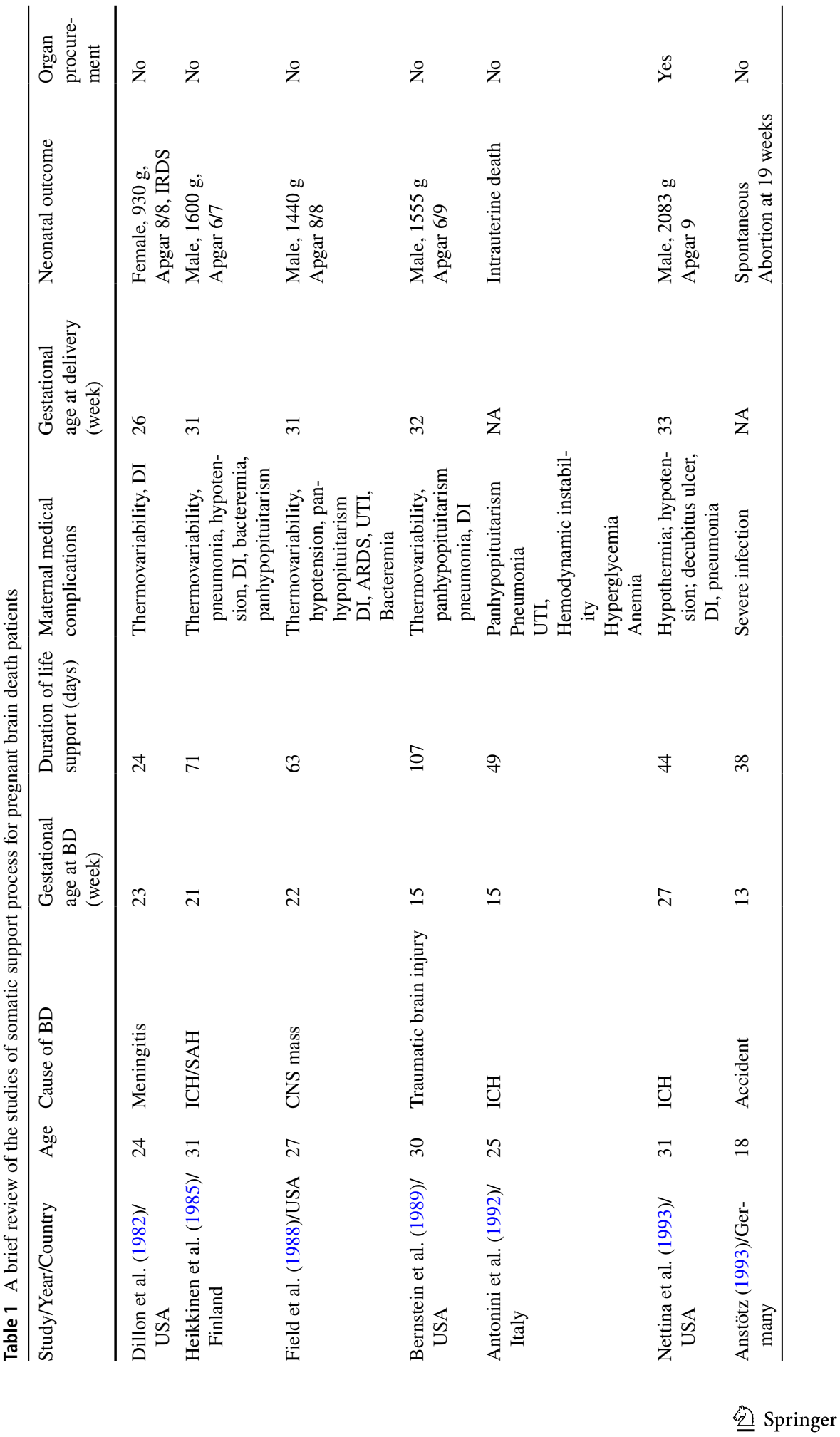




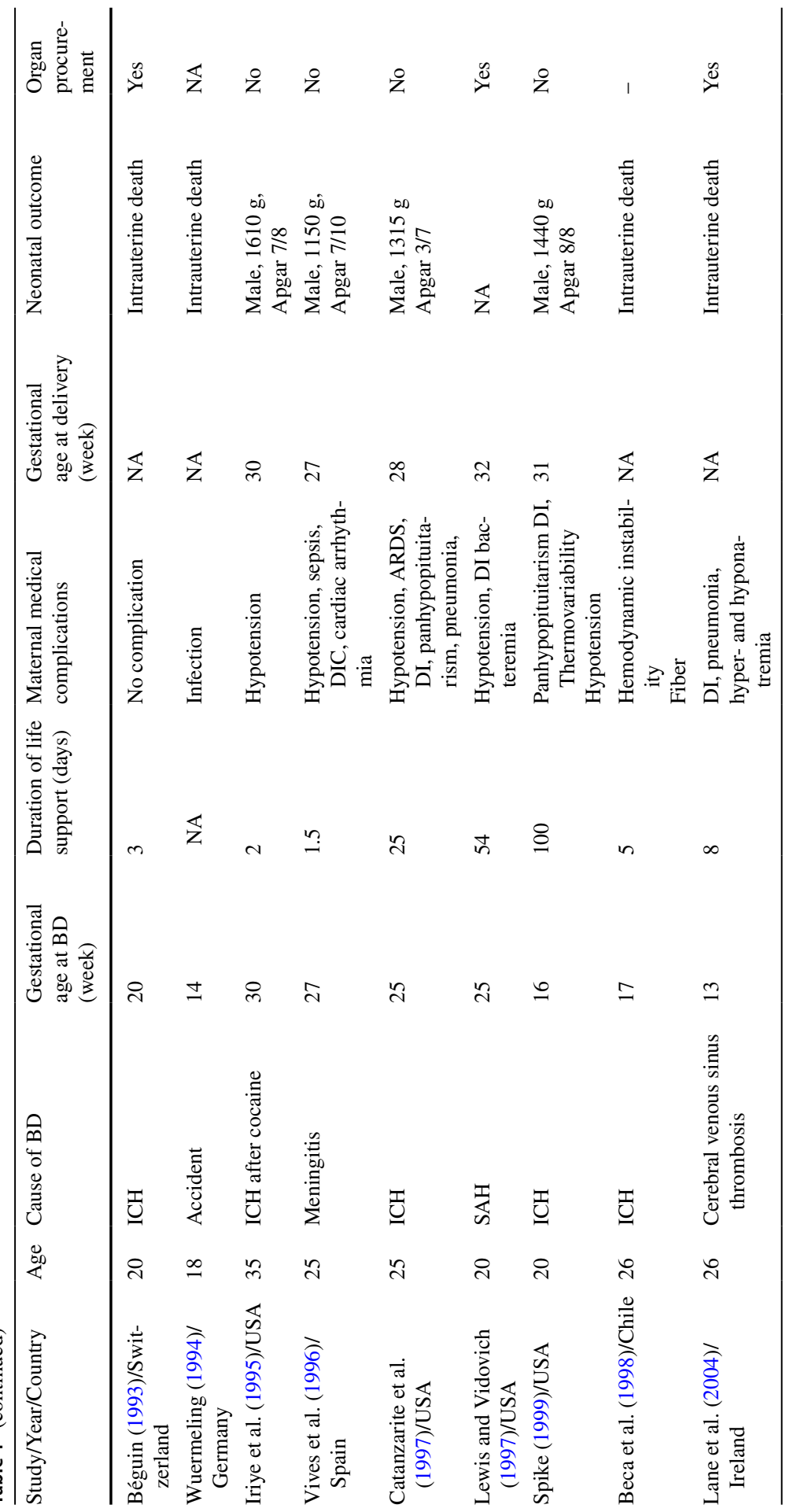




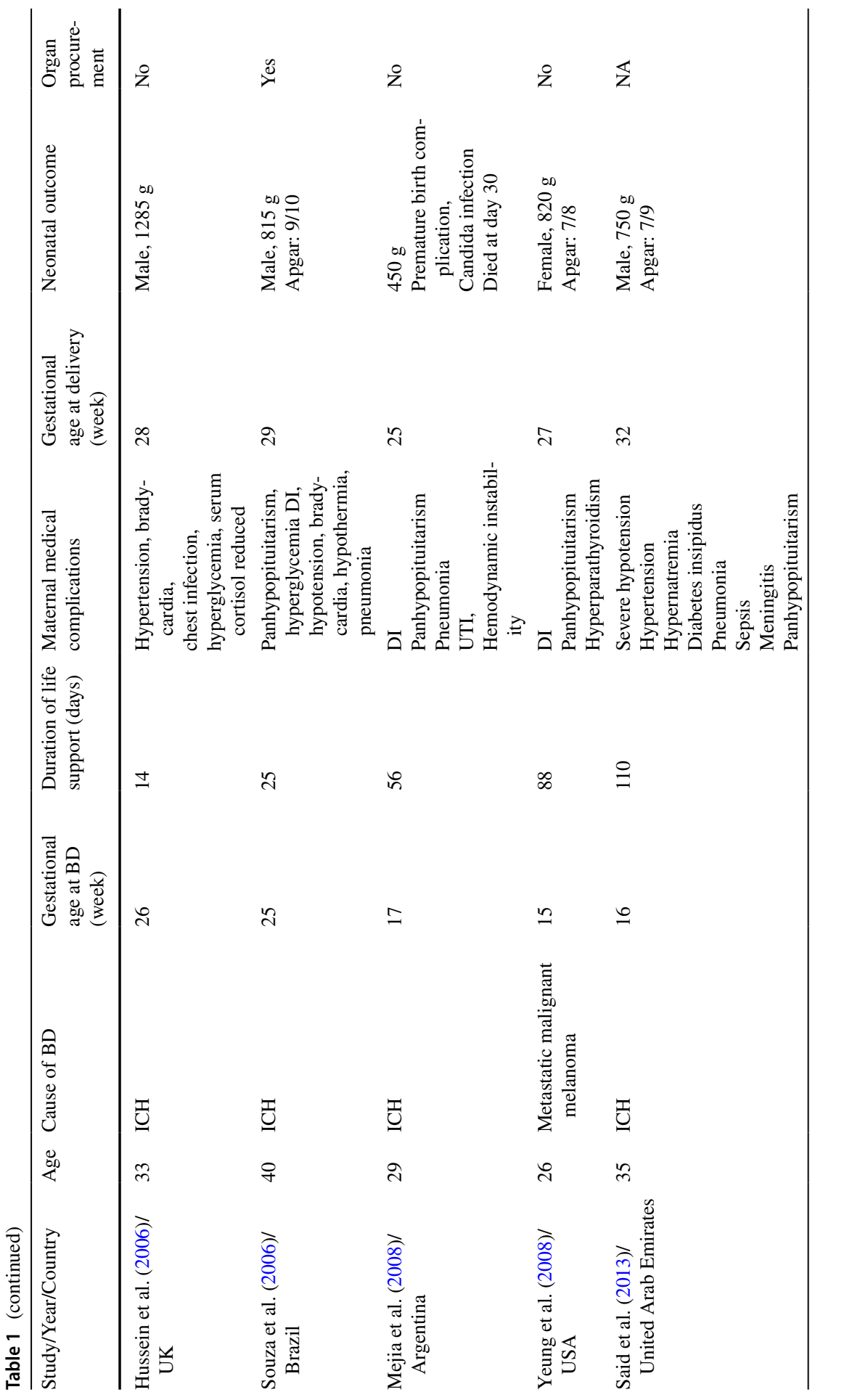




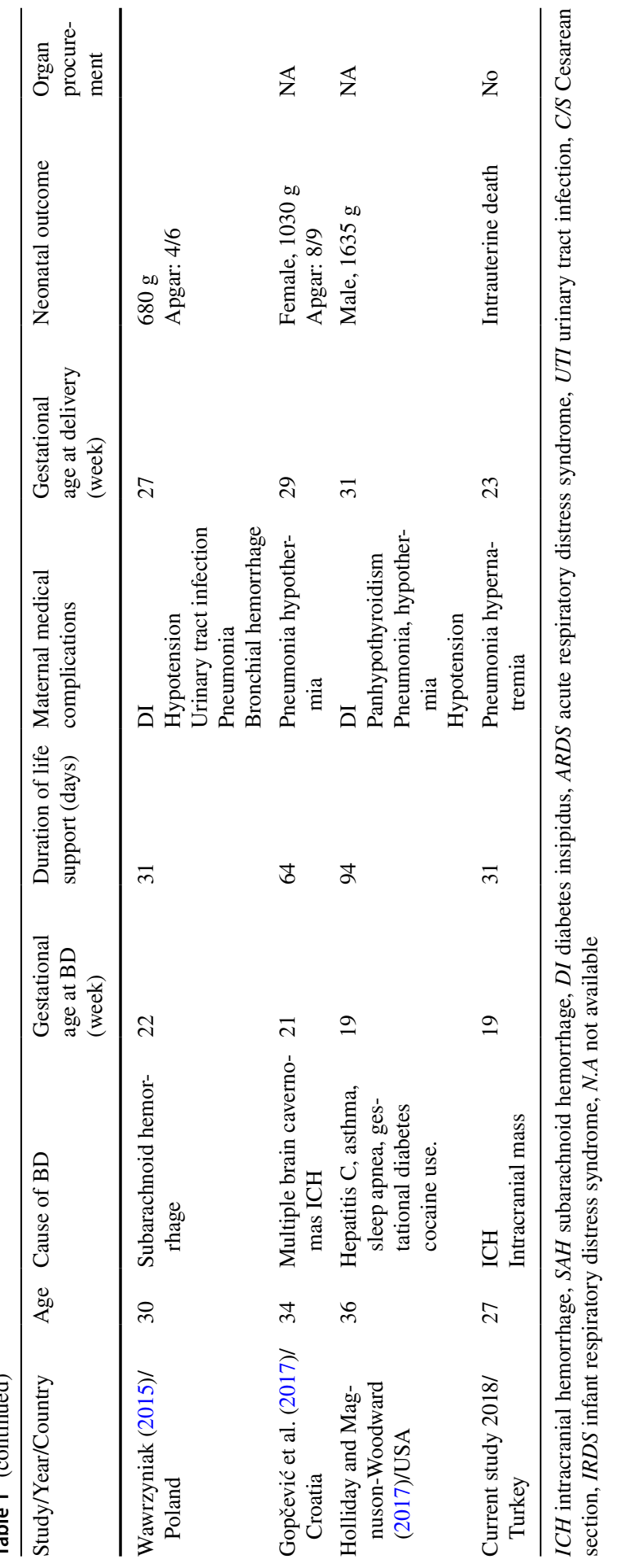




\section{Results}

\section{Case Report}

A female 21-year-old, 19-week pregnant patient with gestational diabetes attended the emergency service with complaints of headache, darkened vision, dizziness and confusion. On admission, Glasgow coma score (GCS) was 11/15, and cranial computed tomography identified intracranial mass and intraventricular hemorrhage. The patient was monitored in the anesthesia reanimation intensive care unit. With worsening general status and GCS score regression to $5 / 15$, the patient was taken for emergency operation by neurosurgery and EVD was inserted. On the postop 3rd day, clinical assessment with suspected BD applied the apnea test and as radiation may threaten the life of the fetus and was contraindicated, the patient had transcranial Doppler ultrasonography performed and BD diagnosis placed. Ultrasonographic assessment confirmed the 19-week fetus was alive, and patient relatives were informed about BD. Initially family members wished to end the pregnancy and support; however, individual interviews were held with family members and they were informed about legal, ethical and medical situations. Family members did not accept somatic support of the mother. Nevertheless, as the process continued, in line with opinions received from an organization with the authority to issue religious rulings, family members changed their decision and reported the mother had planned the pregnancy while healthy and wanted the baby so they wanted to baby to live. With the aim of supporting maternal somatic functions while waiting for fetus resolution, pharmacological support of the cardiovascular system, endocrine replacement treatment (pituitary-thyroid) and enteral nutrition support were administered. When the patient developed hypernatremia, hypotonic fluid replacement and enteral drinking water were administered. When the patient developed a pulmonary infection due to the ventilator, appropriate antibiotherapy began according to culture antibiogram results. The viability of the fetus was monitored with daily fetal ultrasonography. Though the patient survived until the 25 th week, the fetus died intrauterine in the 25 th week. The family members were interviewed again about organ donations. After organ donation was rejected, support was ended. Our cost analysis showed that 9300 dollars extra costs were incurred due to lengthened somatic support treatment of our patient after BD.

\section{Literature Review}

The English-language medical literature published between 1982 and August 2018 was searched using PubMed and Google Scholar. A total of 24 publications were found; however, as the article by Suddaby et al. (1998) about 11 patients developing $\mathrm{BD}$ in the peripartum period did not abide by the inclusion criteria, it was excluded. In this study, four patients developed BD in the early postpartum period, while the other seven patients had earliest BD development in the 30th gestational week (from 30 to 40 gestational weeks). The study did not include any information about 
somatic support treatment after development of BD. As a result, it was not included in the assessment. All other articles were single-patient case reports or literature scans. In our study, a total of 24 pregnant BD cases were identified in the literature. The patients were aged from 18 to 40 years (mean $27.40 \pm 6.06$ years); mean BD development was $20.04 \pm 4.95$ weeks gestation (min-max 13-30). Mean somatic support duration was $42.41 \pm 33.64$ days (2-110 days). In 7 of the 24 patients, the fetus died intrauterine, while 18 babies were born. One of these infants died in the 1 st month due to septic causes. Seventeen infants reached term; however, there was insufficient information about status after birth. The clinicopathological characteristics of the 24 patients are summarized in Table 1. Including our patient, the studies were grouped in terms of live birth of the fetus (Group $1 ; n=18$ ) and intrauterine death of fetus (Group 2, $n=7$ ). The gestational week for BD development in patients included in Group 2 was $15.85 \pm 2.85$ weeks, while this was $21.66 \pm 4.66$ weeks for patients in Group $1(p=0.006)$. The mean intrauterine duration for the fetus after BD development was $22.33 \pm 19.55$ days in Group 2 and $49.11 \pm 35.06$ days in Group 1 $(p=0.092)$. When factors associated with fetus loss are assessed, there appeared to be a serious negative correlation with fetus gestational week when DB developed $(r:-537, p=0.006)$ (Table 2). All of the patients with intrauterine fetus death were 20 weeks or less. In 5 of 15 live births, the date when maternal BD developed was observed to be less than 20 gestational weeks. One of these infants died in the 1st month postpartum. For two patients, somatic support was administered for more than 100 days (107 and 110 days, respectively), and no problem was observed to develop with the infants after birth.

\section{Discussion}

BD occurring during pregnancy is a catastrophic situation for the family and fetus, involving medical, ethical and legal problems without full consensus. Leading these problems is the continuation of medical treatment until maturation of the fetus and extending or ending ventilator support. Due to developments in intensive care conditions and life support technologies, more cases are successfully surviving until birth

Table 2 Evaluation of patients according to the development of intrauterine fetal death

\begin{tabular}{llll}
\hline & $\begin{array}{l}\text { Developing intrauterine } \\
\text { fetal death }(n=7)\end{array}$ & Live birth $(n=18)$ & $p$ \\
\hline Age (year) & $22.00 \pm 3.60$ & $29.50 \pm 5.53$ & 0.003 \\
$\begin{array}{l}\text { Gestational age of fetus when brain } \\
\quad \text { death occurred (weeks) }\end{array}$ & $15.85 \pm 2.85$ & $21.66 \pm 4.66$ & 0.006 \\
$\begin{array}{l}\text { Delivery age (weeks) } \\
\text { Somatic support duration (days) }\end{array}$ & $22.00 \pm 4.24$ & $29.38 \pm 2.37$ & 0.001 \\
\hline
\end{tabular}

Data are expressed as the mean $\pm \mathrm{SD}$, unless otherwise noted. Categorical data are expressed as $n$ (number) and percent (\%). Independent $t$ test (Bootsrap)-Mann-Whitney $U$ test (Monte Carlo)-Fisher's exact test (Monte Carlo) 
occurs and infants maintain viability (Mollaret and Goulon 1959; Dillon et al. 1982). In the literature from 1982 until 2018, there are 24 cases of somatic support administered with the aim of ensuring fetal maturation after development of BD (Dillon et al. 1982; Heikkinen et al. 1985; Field et al. 1988; Bernstein et al. 1989; Antonini et al. 1992; Anstötz 1993; Nettina et al. 1993; Béguin 1993; Wuermeling 1994; Iriye et al. 1995; Vives et al. 1996; Catanzarite et al. 1997; Lewis and Vidovich 1997; Beca et al. 1998; Spike 1999; Lane et al. 2004; Hussein et al. 2006; Souza et al. 2006; Mejia et al. 2008; Yeung et al. 2008; Said et al. 2013; Wawrzyniak 2015; Gopčević et al. 2017; Holliday and Magnuson-Woodward 2017). Of the cases, 18 babies were born and survived the neonatal period (Dillon et al. 1982; Heikkinen et al. 1985; Field et al. 1988; Bernstein et al. 1989, Nettina et al. 1993; Iriye et al. 1995; Vives et al. 1996; Catanzarite et al. 1997; Lewis and Vidovich 1997; Spike 1999; Hussein et al. 2006; Souza et al. 2006; Mejia et al. 2008; Yeung et al. 2008; Said et al. 2013; Wawrzyniak 2015; Gopčević et al. 2017; Holliday and MagnusonWoodward 2017). Including our patient, seven patients had intrauterine fetus death with one baby dying in the 1 st month after birth (Mejia et al. 2008). In our study, the medical problems and legal problems of BD cases occurring in pregnancy accompanied by cases reported in the literature will be debated in terms of ethical values considering religious, human rights and health policies.

\section{Medical Problems}

We believe it is appropriate to discuss the medical problems occurring after BD development under two main headings. The first are maternal medical problems, and the second are medical problems observed in the fetus.

\section{Maternal Medical Problems}

Though health professionals involved in organ transplant encounter many medical problems after BD development, fewer medical problems are encountered as no attempt is made to ensure BD patients survive as long as pregnant BD cases. When examined from this aspect, it may be useful to deal with medical problems that may be encountered in BD patients accepting organ donation to better understand medical problems that may be encountered in pregnant BD patients.

As pregnant $\mathrm{BD}$ patients require specialist branches like neurology, anesthesia, gynecology and endocrinology, they are complex cases requiring a multidisciplinary approach supported by infection and neurosurgery. In our literature assessment, intensive care professionals appeared to begin somatic support with precautions like nutritional support of patients, early tracheostomy opening, dealing with hypothermia and early steroid treatment against development of adrenal failure (Spike 1999; Mallampalli and Guy 2005; Hussein et al. 2006; Souza et al. 2006; Mejia et al. 2008; Yeung et al. 2008; Said et al. 2013; Wawrzyniak 2015; Gopčević et al. 2017; Holliday and Magnuson-Woodward 2017). However, in spite of all precautions, hemodynamic instability led by hypotension, endocrine disorders like adrenal 
failure, diabetes insipidus (DI), panhypopituitarism and hyperparathyroidism, and electrolyte disorders like hypernatremia, hyponatremia, hypo- or hyperpotassemia are some medical problems that may be encountered. Additionally, infectious complications occurring due to lengthened intensive care stay, a variety of invasive instruments and ventilator support are included among life-threatening situations for both mother and fetus (Spike 1999; Mallampalli and Guy 2005; Hussein et al. 2006; Souza et al. 2006; Mejia et al. 2008; Yeung et al. 2008; Said et al. 2013; Wawrzyniak 2015; Gopčević et al. 2017; Holliday and Magnuson-Woodward 2017). Studies by Mallampalli and Guy (2005) dealt with a broad range of maternal complications and satisfactorily summarized the underlying etiologic factors. As stated in this study, many changes observed in the patients occur in the cardiovascular system and actually develop as a response to physiological variations occurring after BD. The initial change is hypertension. This situation occurs linked to cerebral herniation and sympathetic discharge occurring after brain death. Then there is a short normotensive period and then with sympathetic output loss secondary hypotension occurs (Esmaeilzadeh et al. 2010). Hemodynamic instability occurring during this process forms serious risks to the fetus due to disrupted autoregulation of blood flow to the uterus. If not prevented, it may cause fetal hypoxia, neurological injury and even fetal death (Powner 2005). Initial treatment approach is aggressive fluid replacement. Mallampalli and Guy (2005) emphasized the use of crystalloid and colloid combinations for treatment in addition to keeping the patient in left lateral recumbent position. Additionally, in cases resistant to sufficient volume replacement, vasopressor agents may be used. However, it is necessary that the agent used not cause placental vasoconstriction or has minimal side effects like vasopressin (Powner 2005). For our patient, dopamine and norepinephrine were used with this aim.

However, it is not possible to explain hypotension with a single mechanism. Variations occurring in the body after BD are catastrophic and disrupt the hypothalamopituitary axis (Esmaeilzadeh et al. 2010). This situation generally causes panhypopituitarism. Finally, many disorders like diabetes insipidus (DI), adrenal failure, etc., may occur (Gramm et al. 1992). Treatment of these patients involves monitoring electrolyte disorders occurring and administering hypotonic solutions and intravenous, intramuscular ore intranasal desmopressin. Monitoring other hormone levels should not be ignored. Varying serum cortisol and thyroid hormone levels are reported after $\mathrm{BD}$, with the necessity to check hormone levels before replacement emphasized (Howlett et al. 1989). However, resistant hypotension cases in spite of sufficient volume replacement may have adrenal failure in the etiology and replacement of steroids sourced in the adrenal gland should be remembered. In our patient, methylprednisolone treatment was used due to limited transmission through the placenta (Esmaeilzadeh et al. 2010).

During somatic support, many invasive instruments such as central venous catheterization, urine catheter, arterial cannulas, tracheostomy cannulas and intubation cannulas are used with the aim of close monitoring of the mother and baby. This apparatus leaves the patient prone to infection and may cause septicemia. This situation does not just threaten the life of the mother and fetus, and it also causes development of resistant microorganisms. As a result, it needs to be treated aggressively (Bernstein et al. 1989; Esmaeilzadeh et al. 2010; Christensen 2000). Our patient had 
P. aeruginosa and A. baumanii proliferation in two separate tracheal aspirate cultures. Both microorganisms are resistant to many antibiotics and were treated with antibiotics they were susceptible to. In our study, the extra duration of hospital stay and the cost of somatic support treatment administered during this time were calculated as 9300 dollars at the exchange rate of the time.

\section{Situations Related to the Fetus}

Studies have shown the gestational age is inversely proportional to postpartum disability and directly proportional to the baby's chance of survival. In a healthy pregnancy, the minimum duration required for the birth of a normal and healthy baby is 32-34 gestational weeks. Before the 24th gestational week, the survival chance of the newborn is $20-30 \%$, with the possibility of serious neurological disorder $40 \%$. From 24 to 28 weeks, survival increases to $80 \%$, with risk of neurological complications about $10 \%$. From the 32nd week of pregnancy, the survival rate of the fetus rises to $98 \%$, with risk of neurological complications lower than $2 \%$. With this aim, the target is that the fetus reaches the 32nd week (Wood et al. 2000). It has been determined that 24 patients with brain death, about around 35 th years-period, since 1982 according to our literature review (Dillon et al. 1982; Heikkinen et al. 1985; Field et al. 1988; Bernstein et al. 1989; Antonini et al. 1992; Anstötz 1993; Nettina et al. 1993; Béguin 1993; Wuermeling 1994; Iriye et al. 1995; Vives et al. 1996; Catanzarite et al. 1997; Lewis and Vidovich 1997; Beca et al. 1998; Spike 1999; Lane et al. 2004; Hussein et al. 2006; Souza et al. 2006; Mejia et al. 2008; Yeung et al. 2008; Said et al. 2013; Wawrzyniak 2015; Gopčević et al. 2017; Holliday and Magnuson-Woodward 2017). Together with our patient, the fetus died intrauterine in a total of 7 patients (Antonini et al. 1992; Anstötz 1993; Béguin 1993; Wuermeling 1994; Beca et al. 1998; Lane et al. 2004). One baby born in the 25th week died due to infective complications. Studies showed the fetus death rates for BD developing before the 20th week of pregnancy were high and emphasized that administering somatic support is controversial. In our study, all of the patients with fetal death had maternal BD development before the 20th week (Antonini et al. 1992; Anstötz 1993; Béguin 1993; Wuermeling 1994; Beca et al. 1998; Lane et al. 2004). However, of the 18 patients with live birth, five had BD development before the 20th week (Bernstein et al. 1989; Spike 1999; Yeung et al. 2008; Said et al. 2013; Holliday and Magnuson-Woodward 2017). The three patients with longest somatic support duration lived 100 (Spike 1999),107 (Bernstein et al. 1989) and 110 (Said et al. 2013) days. Said et al. (2013) emphasized the need to give a chance to the fetus of mothers developing BD before the 20th gestational week.

\section{Legal Problems}

Currently, though there are legal regulations related to patients developing $\mathrm{BD}$, the situation of pregnant patients developing BD is still not resolved in many countries. The legal rights of the fetus are linked to gestational age and there is a wide range of difference from country to country. Some countries like Iran, Egypt, the Philippines 
and Chile seriously protect the fetus with laws and only allow abortion in situations where the life of the mother is threatened. In Ireland, Venezuela, Nigeria and Mexico, court approval is required. Some countries allow legal abortion until a certain week of pregnancy. As a result, there is no full consensus about BD situations developing in pregnancy from a legal aspect, and there are differences from country to country (Lewis et al. 2016).

The most comprehensive legal regulations related to BD occurring in pregnancy are in the USA. However, here there are some differences between the states (Lewis et al. 2016). Burkle et al. (2015) investigated support of the mother's life in terms of five different categories in US states. In 14 states, somatic support treatment of BD patients is required if there is a chance of live birth of the fetus as stated in the Terminally Ill Act. Four states accepted exceptions where this situation causes physical harm or severe pain to the mother. Some states like California and Hawaii do not have defined regulations related to this situation and leave the decision to the courts if conflict develops (Burkle et al. 2015). Erlinger reported a case developing BD in the USA where pregnancy was ended by the court and could not be continued. However, as stated at the start of this topic, legal regulations have not been made in many countries, with lawmakers in associations involved with organ transplantations required to warn and draw attention to this topic (Erlinger 2017).

\section{Ethical Problems}

One of the important problems with pregnant BD is ethical. The key question for this is whether ensuring somatic support of the mother for the fetus to survive after brain death and in this way to provide lengthened somatic support to the mother is ethical or not (Burkle et al. 2015; Lewis et al. 2016; Erlinger 2017).

One of the two basic dilemmas for this topic is a person's right to autonomy, bodily integrity and a dignified death. The second point is the chance of healthy birth of the fetus, which as we seen in our literature assessment is high enough not to be underestimated (Burkle et al. 2015; Lewis et al. 2016; Erlinger 2017). In the literature, no matter how much the process is emphasized as being experimental especially for early cases developing BD before the 20th week, as shown in our study, nearly one-third of healthy babies born comprised fetuses from cases developing BD before the 20th week (https://kutsal-kitap.net/bible/tr/index .php?id=75\&mc $=1 \& \mathrm{sc}=55$ ). Another topic related to this is that the decision of the mother related to the baby is generally unknown. But as stated in legal topics, there is a legal duration for abortion in many countries and if the mother has passed this duration and not aborted the baby, we believe it must be considered that she wants the baby. However, for pregnancies below the legal abortion limit, this situation may become more complicated. If the thoughts of the mother about this topic are unknown, there should be good communication with family members and after necessary medical and legal information is given the topic should be resolved. Families should be informed and consent obtained especially about developmental or neurological anomalies that may develop in the baby in this situation (Farragher and Laffey 2005; Burkle et al. 2015; Lewis et al. 2016; Erlinger 2017; https://kutsa 
1-kitap.net/bible/tr/index.php?id=75\&mc=1\&sc=55). As emphasized by Farragher and Laffey (2005), with good justification we think, another topic is optimal use of limited resources; in other words, distributed justice. This concept is a situation related to the responsibility of clinicians to society to use the critical care facilities they have access to in a cost-efficient way (https://kutsal-kitap.net/bible/tr/index .php?id=75\&mc $=1 \& \mathrm{sc}=55)$.

\section{Religious Problems}

Though our patient's family members were informed in terms of ethical, medical and legal terms, the critical factor effective in the decision to provide somatic support to the mother was the family obtaining opinions about the baby's survival from an institute with the authority to issue religious rulings. This situation once more emphasizes the importance of religion while people are making important decisions in countries like ours. With this aim, we thought it appropriate to assess the view of a variety of religions about pregnant BD patients in short. Generally, religions make no official statements related to lengthened somatic support of BD cases developing during pregnancy, so we attempted to infer from religious views about abortion. All belief systems see human life as a sacred area requiring protection and respect; as a result, intentional ending of human life is seen as a great sin. Though the Church has no official statement, Catholic teaching requires unconditional respect for the bodily and spiritual integrity of humans (Holy Bible (2018) https://www.wordproject.org/bible $\mathrm{s} / \mathrm{kj} / 40 / 5 . \mathrm{htm})$. According to traditional Jewish belief, the "thou shalt not kill" commandment was among the Ten Commandments given to Moses (Torah (2018) https://www.kingjamesbibleonline.org/Exodus-20-13/). Similarly, in Islam, killing is among the great sins and killing a person is held equivalent to killing all humanity (Quran (2018) http://www.kuranayetleri.net/maide-sures i/yusuf-ali-meali). One of the most important points requiring enlightenment is whether the life within the mother's womb is counted as a separate entity to the mother or when it should be counted as a separate entity or full human. Different approaches are present in the belief systems related to this topic. Common global religions like Islam, Catholicism, Judaism and Buddhism believe that the baby in the mother's womb is not a part of the mother but is a separate entity. At this stage, the topic of when a fetus may be considered a full human is encountered. Islam emphasizes that this boundary is marked by the point where the fetus gains a soul. This is about the 4th month. Early period Christian theologists like St. Augustine considered the fetus in the mother's womb was alive at 60 or 80 days. However, in Catholic teaching, life begins in the mother's womb with conception. Similarly, Buddhism and Hinduism consider the fetus to have a soul from conception. Judaism does not accept the fetus as an individual until birth, emphasizing that the fetus is developing as an individual and requires protection. However, in many religions, there are some points which are exceptions to the general view about abortion, such as danger to the mother's life. 


\section{Conclusion}

Though limited numbers of cases of BD occurring in pregnancy are reported, it is a topic that may occur more commonly involving many problems and requiring important emphasis for families, clinicians and from a legal aspect. Currently the family is the center of this topic, and generally clinicians attempt to resolve this by their efforts. However, especially in countries where social structure is shaped by religious teaching, it should not be forgotten that help may be obtained in managing this process from religious authorities.

Authors' Contribution Concept was contributed by ÖFB, HÖ and DS. FMY, NY and MB contributed to design; supervision was done by ÖFB, HÖ, FMY and DS. ÖFB, HÖ, DS, NY, FMY and MB contributed to resources. Materials were acquired by ÖFB, HÖ, DS and FMY. ÖFB, HÖ, DS, FMY and MB collected and processed the data. Analysis and/or interpretation was carried out by HÖ, NY, MB and FMY. Literature search was done by ÖFB, DS, NY, FMY and HÖ. ÖFB, DS, FMY and NY wrote the manuscript. Critical review was done by HÖ and MB. Diğer/Other have no contribution to be mentioned.

Funding The authors declared that this study has received no financial support.

\section{Compliance with Ethical Standards}

Conflict of interest All authors have no conflicts of interest.

Informed Consent Written informed consent was obtained from patients who participated in this study.

Ethical Approval All procedures performed in studies involving human participants were in accordance with the ethical standards of the institutional and/or national research committee and with the 1964 Helsinki Declaration and its later amendments or comparable ethical standards.

\section{References}

Anstötz, C. (1993). Should a brain-dead pregnant woman carry her child to full term? The case of the "Erlanger baby". Bioethics, 7(4), 340-350.

Antonini, C., Alleva, S., Campailla, M. T., Pelosi, G., Velle, E., \& Verrua, M. (1992). Morte cerebrale e sopravvivenza fetale prolungata. Minerva Anestesiologica, 58, 1247-1252.

Beca, J. P., Wells, W., \& Rubio, R. (1998). Muerte cerebral materna durante el embarazo. Revista Medica de Chile, 126(4), 450-455.

Béguin, F. (1993). Introduction: La mort cérébrale maternelle. In Verhandlungen der Schweizerischen Gesellschaft für Gynäkologie und Geburtshilfe. Berlin: Springer.

Bernstein, I. M., Watson, M., Simmons, G. M., Catalano, P. M., Davis, G., \& Collins, R. (1989). Maternal brain death and prolonged fetal survival. Obstetrics and Gynecology, 74(3), 434-437.

Burkle, C. M., Tessmer-Tuck, J., \& Wijdicks, E. F. (2015). Medical, legal, and ethical challenges associated with pregnancy and catastrophic brain injury. International Journal of Gynecology \& Obstetrics, 129(3), 276-280.

Catanzarite, V. A., Willms, D. C., Holdy, K. E., Gardner, S. E., Ludwig, D. M., \& Cousins, L. M. (1997). Brain death during pregnancy: Tocolytic therapy and aggressive maternal support on behalf of the fetus. American Journal of Perinatology, 14(07), 431-434.

Christensen, B. (2000). Which antibiotics are appropriate for treating bacteriuria in pregnancy? Journal of Antimicrobial Chemotherapy, 46(suppl_1), 29-34. 
Dillon, W. P., Lee, R. V., Tronolone, M. J., Buckwald, S., \& Foote, R. J. (1982). Life support and maternal brain death during pregnancy. JAMA, 248(9), 1089-1091.

Erlinger, L. R. (2017). Guidelines for supporting a pregnant patient with brain death: A case discussion and literature review. Journal of Nursing Education and Practice, 7(8), 86-92.

Esmaeilzadeh, M., Dictus, C., Kayvanpour, E., Sedaghat-Hamedani, F., Eichbaum, M., Hofer, S., et al. (2010). One life ends, another begins: Management of a brain-dead pregnant mother-A systematic review. BMC Medicine, 8(1), 74.

Farragher, R. A., \& Laffey, J. G. (2005). Maternal brain death and somatic support. Neurocritical Care, 3(2), 99-106.

Field, D. R., Gates, E. A., Creasy, R. K., Jonsen, A. R., \& Laros, R. K. (1988). Maternal brain death during pregnancy: Medical and ethical issues. JAMA, 260(6), 816-822.

Gopčević, A., Rode, B., Vučić, M., Horvat, A., Širanović, M., Gavranović, Ž., et al. (2017). Ethical and medical management of a pregnant woman with brain stem death resulting in delivery of a healthy child and organ donation. International Journal of Obstetric Anesthesia, 32, 82-86.

Gramm, H. J., Meinhold, H., Bickel, U., Zimmermann, J., Keller, F., Dennhardt, R., et al. (1992). Acute endocrine failure after brain death? Transplantation, 54(5), 851-857.

Heikkinen, J. E., Rinne, R. I., Alahuhta, S. M., Lumme, J. A., Koivisto, M. E., Kirkinen, P. P., et al. (1985). Life support for 10 weeks with successful fetal outcome after fatal maternal brain damage. British Medical Journal, 290(6477), 1237-1238.

Holliday, S., \& Magnuson-Woodward, B. (2017). Somatic support following cardiac arrest for 90 days leading to a healthy baby boy: A case report. Heart \& Lung: The Journal of Acute and Critical Care, 46(5), 397-400.

Howlett, T. A., Keogh, A. M., Perry, L., Touzel, R., \& Rees, L. H. (1989). Anterior and posterior pituitary function in brain-stem-dead donors. A possible role for hormonal replacement therapy. Transplantation, 47(5), 828-834.

Hussein, I. Y., Govenden, V., Grant, J. M., \& Said, M. R. (2006). Case report: Prolongation of pregnancy in a woman who sustained brain death at 26 weeks of gestation. BJOG: An International Journal of Obstetrics \& Gynaecology, 113(1), 120-122.

Iriye, B. K., Asrat, T., Adashek, J. A., \& Carr, M. H. (1995). Intraventricular haemorrhage and maternal brain death associated with antepartum cocaine abuse. BJOG: An International Journal of Obstetrics \& Gynaecology, 102(1), 68-69.

Lane, A., Westbrook, A., Grady, D., O’Connor, R., Counihan, T. J., Marsh, B., et al. (2004). Maternal brain death: Medical, ethical and legal issues. Intensive Care Medicine, 30(7), 1484-1486.

Lewis, A., Varelas, P., \& Greer, D. (2016). Pregnancy and brain death: Lack of guidance in US hospital policies. American Journal of Perinatology, 33(14), 1382-1387.

Lewis, D. D., \& Vidovich, R. R. (1997). Organ recovery following childbirth by a brain-dead mother: A case report. Journal of Transplant Coordination, 7(3), 103-105.

Mallampalli, A., \& Guy, E. (2005). Cardiac arrest in pregnancy and somatic support after brain death. Critical Care Medicine, 33(10), 325-331.

Mejia, R., Badariotti, G., De, B. D., Ridruejo, O., \& O'Flaherty, E. (2008). Brain death in a pregnant woman and fetus survival. Medicina, 68(6), 447-452.

Mollaret, P., \& Goulon, M. (1959). Le coma dépassé. Rev Neurol (Paris), 101, 3-15.

Nettina, M., Santos, E., Ascioti, K. J., \& Barber, M. A. (1993). Sheila's death created many rings of life. Nursing, 23(3), 44-48.

Powner, D. J. (2005). Variables during care of adult donors that can influence outcomes of kidney transplantation. Progress in Transplantation, 15(3), 219-225.

Said, A., Al Jundi Amer, U. R. M., Dirar, A., \& Faris, C. (2013). A brain-dead pregnant woman with prolonged somatic support and successful neonatal outcome: A grand rounds case with a detailed review of literature and ethical considerations. International Journal of Critical Illness and Injury Science, 3(3), 220.

Souza, J. P., Oliveira-Neto, A., Surita, F. G., Cecatti, J. G., Amaral, E., \& e Silva, J. L. P. (2006). The prolongation of somatic support in a pregnant woman with brain-death: A case report. Reproductive health, 3(1), 3.

Spike, J. (1999). Brain death, pregnancy, and posthumous motherhood. Journal of Clinical Ethics, 10, 57-65.

Suddaby, E. C., Schaeffer, M. J., Brigham, L. E., \& Shaver, T. R. (1998). Analysis of organ donors in the peripartum period. Journal of Transplant Coordination, 8(1), 35-39. 
Vives, A., Carmona, F., Zabala, E., Fernandez, C., Cararach, V., \& Iglesias, X. (1996). Maternal brain death during pregnancy. International Journal of Gynecology \& Obstetrics, 52(1), 67-69.

Wawrzyniak, J. (2015). Continuation of pregnancy in a woman with critical brain injury. Anaesthesiology Intensive Therapy, 47(1), 40-44.

Wood, N. S., Marlow, N., Costeloe, K., Gibson, A. T., \& Wilkinson, A. R. (2000). Neurologic and developmental disability after extremely preterm birth. New England Journal of Medicine, 343(6), 378-384.

Wuermeling, H. B. (1994). Brain-death and pregnancy. Forensic Science International, 69(3), $243-245$.

Yeung, P., Jr., McManus, C., \& Tchabo, J. G. (2008). Extended somatic support for a pregnant woman with brain death from metastatic malignant melanoma: A case report. The Journal of MaternalFetal \& Neonatal Medicine, 21(7), 509-511.

Publisher's Note Springer Nature remains neutral with regard to jurisdictional claims in published maps and institutional affiliations.

\section{Affiliations}

\section{Ömer Faruk Boran ${ }^{1}\left[\right.$ - Fatih Mehmet Yazar $^{2}\left([) \cdot\right.$ Murat Bakacak $^{3}([)$.

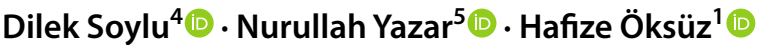

Fatih Mehmet Yazar

fmyazar@ksu.edu.tr

Murat Bakacak

muratbakacak46@gmail.com

Dilek Soylu

soyludilek2009@gmail.com

Nurullah Yazar

nurullah_yazar@hotmail.com

Hafize Öksüz

drhoksuz@hotmail.com

1 Department of Anesthesiology and Reanimation, Sütçü Imam University School of Medicine, Kahramanmaraş, Turkey

2 Department of General Surgery, Sütçü Imam University School of Medicine, Kahramanmaraş, Turkey

3 Private Vatan Hospital, Kahramanmaraş, Turkey

4 Sütçü Imam University School of Medicine, Kahramanmaraş, Turkey

5 Faculty of Divinity, Ankara University, Ankara, Turkey 